Citation: Journal of Paediatric Dentistry DOI: 10.1111/ipd.12302

http://onlinelibrary.wiley.com/journal/10.1111/(ISSN)1365-263X

Do dentists use validated child dental anxiety measures in clinical practice? A mixed methods study

H. Buchanan, H. Alshammasi, P. Ashley

\title{
Dentists' use of validated child dental anxiety measures in clinical practice: A mixed methods study Summary
}

Background: Assessing anxiety is an important part of the assessment of a child presenting for dental treatment. However, the use of dental anxiety scales in practice is not well-documented.

Aims: To introduce child dental anxiety scales, and to monitor the extent to which dentists used them; to explore the experience and views of dentists regarding anxiety assessment.

Design: A mixed-methods design was employed.

Method: A protocol for child anxiety assessment was introduced to pediatric dentists in Eastman Dental Hospital. After 6 months, 100 patient files were audited to examine compliance with the protocol. Fourteen dentists were interviewed to explore their experience and views regarding anxiety assessment.

Results Only five patients were assessed using the scales. Thematic analysis of the dentist interviews revealed three themes: 'Clinical observations and experience: The gold standard'; 'Scales as an estimate or adjunct' and 'Shortcomings and barriers to using scales'.

Conclusions: The dentists in our study did not use anxiety scales, instead they rely on their own experience/judgment. Therefore, scales should be recommended as an 
adjunct to judgment. Brief scales are recommended as clinicians lack time and expertise in administering anxiety questionnaires. Advantages of using scales and hands-on experience could be incorporated more in undergraduate training.

Keywords: Anxiety Scales, ACDAS, Facial Image Scale, Child Anxiety 


\section{Introduction}

Dental anxiety is a common problem that develops mostly in childhood and adolescence $(1,2)$. Although prevalence reports vary, it is indicated that approximately half of children report low to moderate dental anxiety and between $10 \%$ and $20 \%$ report high levels of dental anxiety (3). Anxious children are more likely to have caries $(4,5)$, and are more likely to report pain during dental procedures (6). They are one of the key groups that are referred to secondary dental services from general dentists, resulting in longer periods before dental treatment can be delivered (7). In addition, $47 \%$ of paediatric dentists feel that anxious children require special techniques for them to be managed (8).

The detection and effective management of dental anxiety is an important part of the dental practitioner's role (9). A number of validated dental anxiety questionnaires are available to help practitioners reach an accurate assessment of dental anxiety in patients (10). These measures have a number of advantages, for example, they can help highlight dentally anxious patients, or patients who are anxious of a particular procedure or dental context. This information can then be used to inform appropriate management techniques as well as identifying that these patients may take longer to treat. Communication is key when treating anxious patients and routinely administering dental anxiety scales to new patients can act as a tool for communication between child, parent and dentist (11). Completing questionnaires and scales can act as a vehicle for children to communicate their worries and their needs - discussion of the responses on the questionnaire can help build rapport and trust with the anxious child.

The use of dental anxiety scales in research is well reported, with systematic reviews demonstrating their strengths and limitations as well as psychometric properties $(10,12)$. However, their use in dental clinical practice is not well-documented. The evidence that is available shows that they are not routinely utilised. In a 2001 survey investigating the use of anxiety scales by 328 UK practitioners \{who listed themselves as interested in treating dentally anxious patients\}, only 46 practitioners (17\%) used child dental anxiety assessment questionnaires (13).

Therefore, our mixed-method study had two key aims. Firstly, as previous research has indicated very limited use of anxiety scales in clinical practice, we aimed to introduce and promote the routine use of state and trait anxiety scales, and to monitor the extent to 
which the dental staff used these scales over a 6-month period in a busy Paediatric Department in the Eastman Dental Hospital (EDH) in London, UK. As children are routinely referred to secondary care for anxiety management, we felt it particularly pertinent to gauge formal anxiety assessment in this context. Second, we aimed to explore qualitatively the experience and views of EDH dentists regarding anxiety assessment in clinical settings.

\section{Setting}

The research took place in the Paediatric Department at the Eastman Dental Hospital which is part of the University College London Hospitals NHS Foundation Trust. The hospital is one of the largest secondary/tertiary dental care providers in North London with 85,000 outpatients annually attending the dental hospital. The department is a specialist dental unit that provides dental treatment for children up to the age of 18 years. Annually almost 2600 new patients are referred and there are over 5800 dental appointments \{with almost 1000 general anaesthetic procedures annually\}.

\section{Materials and Methods}

\section{Design}

A mixed-methods design was employed. A protocol for child anxiety assessment was introduced to paediatric dentists in Eastman Dental Hospital. We conducted an audit of patient notes to determine the extent to which the dentists were following this protocol and using scales in practice. We used a qualitative approach ssemi-structured interviews $\}$ to explore in-depth the views and experience of dentists in terms of anxiety assessment in clinical practice.

\section{Measures}

Self-report child anxiety scales are mainly divided into two main categories. "State" anxiety scales are indicators of how the child is feeling at that time in the dental clinic (or how they are feeling about what is about to happen e.g. treatment); they can also be used to assess immediate retrospective anxiety \{i.e. asking a child at the end of their treatment session how generally they felt during it\}. "Trait" measures are those scales that give a more 'global' or comprehensive measure of anxiety (that can be employed out of the dental setting). They are usually longer scales that ask the child how they 
would feel across a variety of dental contexts or procedures. Our final protocol included a mix of both state and trait anxiety scales, across dental appointments (see below).

We employed the Abeer Cognitive Dental Anxiety Scale (ACDAS which was developed within the Paediatric Department at the Eastman Dental Hospital. The scale is formed of three components, the first two are the Dental and Cognitive components which are completed by the child. The first part asks 'How do you feel about..' followed by thirteen items related to the dental visit including procedures and sensations (sight, hearing, taste, smell and touch). Example questions include 'A dentist checking your teeth with a mirror' and 'Having a "pinch" feeling on the back of your hand'. Children respond to the questions on a 3 face response set (reflecting 'happy', 'okay' and 'scared' faces). The second part has three questions related to the child's dental-related cognitions/beliefs (losing control, embarrassment and self-confidence); children respond either 'yes' or 'no' to each question. The third part provides the opportunity for the child's parent/legal guardian to establish whether the child has had previous dental treatment ('Has your child had previous dental treatment?' (yes/no) and expectations of their behaviour at that visit 'How do you expect your child's behaviour today?' (Happy/ OK/Scared). Finally, at the end of the visit, the dentist is asked to tick 'What is your rating for the child's behaviour?' on a three-point scale (Happy/ OK/Scared).

In the initial validation study (14), this scale demonstrated good concurrent, discriminative and convergent validity in children of 6 years and above. Cronbach's alpha $(\alpha)$ was 0.90 , which indicated excellent internal consistency. It takes approximately 5 minutes to complete.

The Facial Image Scale (FIS) $(15,16)$, is a one-item state measure of dental anxiety; it comprises 5 faces and the child is asked to simply point at the face they feel most like at that time ( 1 = happiest face; $5=$ unhappiest face). It is quick and easy to administer and provides immediate 'state' feedback to the clinician (although it does not necessarily inform the dental practitioner, or the researcher, regarding what the child patient is anxious about). The FIS has established concurrent validity and can be used with children from the age of three years (15). No reliability estimates are available for this measure as it is a one 'item' state measure. It is regularly employed in research studies (17-19).

\section{Procedure}




\section{Implementing the routine anxiety assessment protocol}

\section{Abeer Cognitive Dental Anxiety Scale (ACDAS) - first and final appointment}

Within the outlined protocol, all EDH paediatric dentists were asked to request that children of age 5 and over complete the ACDAS at the pre-treatment appointment and on their final dental visit. Taking a measure of their trait anxiety at the beginning of treatment can help alert practitioners to procedures and cognitions (such as fear of losing control) that child patients may be specifically anxious about. The ACDAS was handed to the parent/child by the front reception desk when the patient arrived for their first screening dental appointment. The protocol outlined that it should be completed in the waiting room before any treatment. The child patient could complete their part on their own or with help from their parent (depending on age); the parent completed their part (two questions) on the ACDAS and were asked to hand it over to the dentist at the start of the appointment. If for any reason the front desk did not provide the ACDAS, the dentists were asked to give them a copy (spare copies were made available) and have the child/parent complete it at this point. The dentist was asked to complete their oneitem rating of the child's behaviour on the ACDAS at the end of the appointment.

Employing the ACDAS at the end of all their treatment at the hospital enables us to see if there has been any difference in trait dental anxiety from the first/last appointment. Therefore, the ACDAS was implemented following the same procedure on the final appointment.

\section{Facial Image Scale - all other appointments}

The Facial Image Scale (FIS) was administered at all other appointments. The rationale for this is that the FIS is very quick to administer and score (typically less than 30 seconds) and gives information about how the child is feeling about the appointment/treatment that day. We advised the dentists to use this scale with all children from three years and over. They were asked to show the FIS to the child on the dental chair and to record their response by ticking their chosen face.

\section{$\underline{\text { Recording and filing of anxiety scales/discussions }}$}


Dentists were asked to record on the protocol sheet whether they did or did not discuss the child's anxiety score(s) in any way \{along with any other relevant notes\} and to file all the scales in the patient files. 


\section{Setting out of the protocol}

The researchers set out the protocol for the use of anxiety scales to dental staff. This was carried out in various ways. First, dental staff were given a presentation by a health psychologist (HB) outlining the proposed protocol and the advantages of using anxiety scales in clinical practice. Staff were encouraged to comment on the protocol and to discuss any problems envisaged with carrying this out. There were no suggestions from staff regarding changes to the protocol. Staff were reminded to use the scales via emails and during monthly staff meetings by the first author.

\section{Experience and views of child anxiety assessment: Dentist interviews}

We used in-depth interviewing to explore in detail the experience and views of dentists regarding child anxiety assessment in clinical settings. Nineteen dentists were approached via email invitation that included a PDF attachment of the study information sheet. The list of dentists approached included consultants, specialists, community dentists, postgraduates, Dental Core Trainees (DCT) and Paediatric Speciality Trainees (STRs); all working at the paediatric department at Eastman Dental Hospital. We recorded whether they worked full-time or part-time at the department. Fourteen dentists agreed to take part while 5 chose not to due to lack of time.

Interviews were conducted by the first author \{a paediatric dentist\} and lasted between 38 - 96 minutes. Most interviews were conducted on a side office located in the Paediatric Department at the Eastman Dental Hospital; one was conducted in the dentist's office located at their community dental centre and one was conducted in the dentist's home. Questions aimed to explore: Participants' knowledge of anxiety scales; their current methods of assessing anxiety; anxiety scales weaknesses/strengths in their view; how dentists think anxiety scales should be; are anxiety scales be helpful or not; facilitators/barriers to using anxiety scales. All interviews were recorded using a digital Dictaphone and then transcribed verbatim.

\section{Ethical Considerations}

Ethical approval was granted by NRES Committee London - London Bridge \{REC reference number: 12/LO/1454\} and approval from the Research and Development Director of UCLH - NHS Foundation Trust was also granted. The first author explained 
consent and withdrawal procedures to participants and attained their consent through signing of a consent form. All transcripts were anonymised.

\section{Data analysis}

In order to evaluate the extent to which dentists were following the protocol, we reviewed 100 randomized \{using a computer program\} patient files 6 months after introducing the anxiety scales to the department. Following this, dentist's interviews were audiorecorded, transcribed and thematically analysed following the six steps process outlined by Braun and Clarke (20). The first author read all of the participant responses in full, several times, to ensure familiarity with the data. Careful consideration was given to the context of the participant's responses to ensure that their full ideas and thoughts are reported and unchanged. Additionally, to ensure rigour the thematic interpretation was discussed between all the authors.

\section{Results}

\section{Audit of anxiety scale protocol}

Results showed very poor compliance with the protocol. Only 4 patients were assessed by the FIS and only during their first dental visit; no record was found for children being assessed on any subsequent visits. Only 1 patient out of 89 who should have been administered the ACDAS aged 5 and above\}, was actually administered it.

\section{Experience and views of child anxiety assessment: Dentist interviews}

\section{Participants}

The participants were asked about their dental training (see Table 1). Participants originated from 7 different countries; UK, Saudi Arabia, Egypt, India, Greece, Malaysia and Brazil, with variation between the years of graduations of undergraduate school (between 1979 and 2009). They were also in different stages of specialty training; Dental Core Trainees, specialist postgraduate training, specialist dentist and consultant. All of the dentists practiced in different clinics, private practices, community and speciality dental hospital. All were either full time or part time dentists working in the Eastman Dental Hospital. 
Despite different dental school backgrounds and years of graduation, none of the dentists reported being exposed to, or taught about, dental anxiety scales during their undergraduate dental training. However, some dentists had a limited knowledge as a result of doing a research project during their postgraduate training. Three key themes were identified in the data, which will be discussed in turn.

- Table 1 about here -

\section{Clinical observations and experience: The gold standard for anxiety assessment in practice}

It was clear from the interviews that the pervasive view of the participants was that clinical experience and observations were how they identified anxious patients. Indeed, all of the dentists indicated that this is how they determine if a child is anxious or not. This method is considered to be more trustworthy than self-report anxiety scales:

Interview 14: "I feel, from my experience and from my meeting the child etc., I will be able to assess that anyway"

These opinions were shared among all of the dentists, regardless of their dental school background and years of graduation. However, there was some indication that practitioners with less experience, could benefit from using scales:

Interview 5: "I think I'm a better judge, I don't need the tools, but I think maybe people who don't have the, I'm not saying l've got amazing experience, but people who haven't seen as many people or children maybe could use them and it would be quite helpful" Participants reported that they look for obvious child behaviour such as crying, screaming, clutching to their parents and refusing to get on the dental chair:

Interview 4: "They don't want to leave their mother, they don't want to sit in the chair, sometimes they cry, sometimes they refuse to open their mouth" 


\section{Shortcomings and barriers to using scales in clinical practice}

The dentists highlighted many shortcomings and barriers to using child anxiety scales in clinical practice. Many dentists questioned the reliability and validity of anxiety scales as a source of information, or as a tool that can distinguish between anxious/non anxious children. Several reported that they felt they were not practical, as they require time to complete, and some scales require additional scoring. Moreover, some dentists were concerned regarding the terminology used in these scales as they felt this may precipitate anxiety rather than detecting it.

Dentists felt anxiety scales should be straightforward, easy to use and require no additional scoring. Some dentists felt that existing child anxiety scales were too complicated for children:

Interview 5: "I don't personally think they're the greatest tools, I think it's very difficult for a child to use them ...but it has to be from the child, you can't use the parents, I don't think that's a good judge either"

Indeed, even using faces in a scale was seen as needing modifications for children:

Interview 6: "The five scale, you know the five faces... I think there should only be three, happy sad or nothing"

A scale which integrated a form of calibration like a ruler was mentioned by several dentists in order to obtain a score, but not to precipitate anxiety.

Interview 13: "Any scales that I was taught are the best are the ones that the child has got a ruler that you measure because they can see what it means."

Many of the dentists raised concerns about children as reliable indicators of their own anxiety. They felt that a response from a child on an anxiety scale would be 'meaningless' because either they would not understand the question being asked or they would have answered it in a random fashion due to being bored or anxious. They also highlighted that children look for the 'right' answer to impress the dentist or parent. 
Most dentists indicated that lack of time while running a busy clinic was a significant barrier to using anxiety in the clinic. Lack of experience of using scales, was also highlighted. Indeed, one participant illustrated the interaction between both factors and indicated that she might have considered using them otherwise:

Interview 1: "I haven't got enough experience with using the scales because of the time factor, so if I have enough time I would consider them, yeah."

The majority of the dentists felt that anxiety scales are mainly designed for research and they cannot be implemented in clinical settings.

\section{Scales as an 'estimate' or adjunct}

There were some dentists who highlighted where child anxiety scales could be useful, but these were not as either a stand-alone measure, or providing a precise indication.

For example, some of the dentists considered the scales could be of use as an initial 'estimate' of the child's anxiety:

Interview 8: "Okay, I would say that the scales would assess the level of anxiety of the patient and it reflects how they're feeling, it might not be an exact reflection, however, it's close enough it gives us an estimate to know which category this child is in so it just gives you, are they really very anxious"

Scales were also seen as a possible addition to the clinicians' own judgment, though there is still need the need to take into account other contextual, clinical and patient factors:

Interview 6: "That scale doesn't take into account any individual factors of the patient, of the environment, of the treatment that needs to be done, of what's gone on, of what stage, if they're new, follow-up, whatever, so I think it's a tool but it has to be used with all the other factors together"

Few dentists acknowledged that anxiety scales might have a role in clinical settings. However, one participant provided a scenario in which they can be considered useful: 
Interview 2: "So if I open up the notes and see "oh this patient is actually very happy" so I presume that my first expression to the patient is actually, I presume that "oh this patient going to be cooperative"

\section{Discussion}

There is a lack of data on both the use of anxiety scales for children in clinical practice, and dentists' views and experience of these. In our study we aimed to introduce and promote the routine use of anxiety scales in a busy London dental paediatric dental Unit, and to monitor the extent to which the dental staff used these scales. We also set out to explore qualitatively the experience and views of these dentists regarding anxiety assessment in clinical settings.

Our findings from the anxiety scale audit were striking. There was very poor compliance to the protocol introduced to the EDH dentists for routine assessment of child anxiety. Importantly, the follow-up interviews provided valuable data on why there was poor uptake of anxiety measurement. One key explanation may be the lack of knowledge and understanding of scales. Despite different dental school backgrounds and years since graduation, all of the dentists reported that they had not been taught about dental anxiety scales during their undergraduate dental training. This may be a recall bias \{they may have been included in teaching but not remembered\}, or it may be the case that these were indeed not included in the curriculum.

It is now a requirement that UK undergraduates are taught about management and assessment of dentally anxious patients in the Preparing for Practice guidelines (21). However, the extent to which courses cover anxiety scales (and the detail in which they go into, e.g., the advantages of formal measurement) is unclear. Therefore, there may be scope for highlighting anxiety scales (for children and adults) in a more formal capacity in undergraduate training in the UK (and other countries). Lack of experience of using scales, was also highlighted as a reason for lack of use. Therefore, training at undergraduate level could involve demonstrating how to use scales, how to score them and hands-on experience of using them in the clinic. 
The participants were mostly negative about using dental anxiety scales in clinical practice. Many dentists questioned the reliability and validity of anxiety scales as a source of information, or as a tool that can distinguish between anxious/non anxious children. Although psychometric properties have been established and published (10), the dentists did not appear convinced of their role in practice. This could again be highlighted in undergraduate training. One of the key barriers was the concern that the items in the scales can make the children more anxious, or more generally that by assessing anxiety it can cause anxiety. This was a worry that was shared across the participants. However, it has been established in the literature that the effect can be quite the opposite. Several studies with adults have shown assessment and discussion can actually reduce patients' anxiety $(22,23)$. Future research should seek to assess if this is also the case in a child population. If so, this should be highlighted to dentists.

A lack of time was highlighted by participants as an important barrier to using scales. This is perhaps unsurprising, as this is a busy clinic with a large number of referrals. We attempted to combat this by including a longer measure only at the first and last appointment, and the FIS across appointments. It may be the case that just using the FIS may help uptake in the future and may standardise the protocol, with one less thing to remember to do. It would also overcome the concern that the participants had about lack of expertise in scoring (the FIS incorporates just a row of faces). Future research should evaluate this.

All of the dentists indicated that they rely on their experience and observations to determine if a child is anxious or not. They mostly look for behavioural signs of anxiety such as crying and not wanting to be separated from parents. There is evidence for the validity of clinical observations (23) but relying solely on these may be problematic; dental anxiety is multidimensional (which can involve cognitive, behavioural and physiological components) and can manifest itself differently across children. There can be a lack of synchrony between behaviour and internal psychological states,(24), that is not all child patients show behavioural signs of anxiety. Moreover, even when a child does display their anxiety in behaviour this may appear counter-intuitive - for example, they may 'freeze' in the dental chair which can present as co-operation. Initial validation of the Facial Image Scale \{FIS\} showed only moderate agreement between dentists and children on the same scale prompting the authors to conclude that it is still important to 
have children reflect their own anxiety in the dental clinic on a validated scale, rather than solely relying on clinical observations (16).

As the participants highlighted that scales are most useful as an 'adjunct' to their own judgment, we suggest this is the way to incorporate anxiety scales into practice recognising that they are not 'stand-alone' tools, but are to be used in conjunction with dentists' own experience. In addition, as some of the participants highlighted, clinicians who are not so experienced may find that scales help them develop their clinical skills in identifying highly anxious participants (or indeed distinguishing between anxious patients and those with behaviour management issues).

Anxiety management should also be concerned with preventing the escalation of anxiety. Thus it is also useful to use scales to highlight children with low or moderate levels of anxiety (and who may not display typical 'anxiety behaviours'). Efforts are still needed to promote positive experiences for these children, to reassure them, and address any concerns they have in order to prevent their anxiety from increasing further in the future. It has been identified that different levels, and consequently the management of dental anxiety, involves both assessment and proportionate intervention (25). Those with high levels may need more structured psychological and pharmacological interventions. Using scales across treatment appointments can help monitor whether interventions are being effective.

We attempted to get the dentists at EDH to 'get on board' with the routine anxiety assessment by highlighting the advantages of scales, and by making the protocol easy to follow. We also asked for feedback to incorporate into the protocol to try to overcome any barriers before we started. Clinicians were also reminded at meetings and in emails. There are several key aspects we can take from this. First, it may the case that participants had reservations about the protocol but did not voice these. A follow-on smaller group discussion may have tapped into these concerns better. It may also have helped to have had discussions throughout the 6 month's audit period which may have addressed some of the barriers highlighted in the interviews earlier. Now we have this data we can help incorporate this for future attempts at establishing routine anxiety assessment.

\section{Limitations of the study}


It should be acknowledged that we sampled from only one dental hospital in London, therefore we cannot establish whether these audit findings and views would also be indicative of secondary care dentists more widely.

\section{Conclusion}

Anxiety still poses a significant problem for the practice of dentistry, so detecting and assessing dental anxiety among child patients is necessary. The dentists participating in our study did not use formal scales and are generally happy and satisfied with their current method of assessment where they rely on their experience and judgment; there is generally a feeling that additional tools are not needed. It may be the case that scales should be highlighted as an adjunct to judgment, and a tool to help those who lack experience. Brief measures are recommended in clinical practice as time and expertise are highlighted as barriers. The advantages of using scales fincluding an aid to communication\}, and hands-on experience could be included more in undergraduate training.

\section{Why this paper is important to paediatric dentists:}

- $\quad$ Anxiety still poses a significant problem for the practice of dentistry, so detecting and assessing dental anxiety among child patients is necessary.

- Brief, validated anxiety scales may be a useful adjunct to clinical observations, clinical judgment and a tool to help those who lack experience.

- $\quad$ Advantages of using scales (including an aid to communication), and hands-on experience could be included more in undergraduate training. 


\section{References:}

1. Locker D, Thomson WM, Poulton R. Onset of and patterns of change in dental anxiety in adolescence and early adulthood: a birth cohort study. J Dent Res. 2001;18(2):99-104.

2. Locker D, Liddell A, Dempster L, Shapiro D. Age of onset of dental anxiety. J Dent Res. 1999;78(3):790-6.

3. Taani DQ, El-Qaderi SS, Abu Alhaija ES. Dental anxiety in children and its relationship to dental caries and gingival condition. Int J Dent Hyg. 2005;3(2):83-7.

4. Berggren U, Meynert G. Dental fear and avoidance: causes, symptoms, and consequences. J Am Dent Assoc. 1984;109(2):247-51.

5. Nicolas E, Bessadet M, Collado V, Carrasco P, Rogerleroi V, Hennequin M. Factors affecting dental fear in French children aged 5-12 years. Int J Paediatr Dent. 2010;20(5):366-73.

6. Mustafa O, Parekh S, Ashley P, Anand P. Post-operative pain and anxiety related to dental procedures in children. Eur J Paediatr Dent. 2013;14(4):289-94. 7. Harris RV, Pender SM, Merry A, Leo A. Unravelling referral paths relating to the dental care of children: a study in Liverpool. Prim Dent Care. 2008;15(2):45-52.

8. Crossley ML, Joshi G. An investigation of paediatric dentists' attitudes towards parental accompaniment and behavioural management techniques in the UK. Brit Dent J. 2002;192(9):517-21.

9. Schuller AA, Willumsen T, Holst D. Are there differences in oral health and oral health behavior between individuals with high and low dental fear? Community Dent Oral Epidemiol. 2003;31(2):116-21.

10. Porritt J, Buchanan H, Hall M, Gilchrist F, Marshman Z. Assessing children's dental anxiety: a systematic review of current measures. Community Dent Oral Epidemiol. 2013;41(2):130-42.

11. Buchanan H. Acquisition and measurement of dental anxiety. Social Sciences \& Dentistry. 2002;2(1):20-4.

12. Aartman IH, van Everdingen T, Hoogstraten J, Schuurs AH. Self-report measurements of dental anxiety and fear in children: a critical assessment. ASDC $J$ Dent Child. 1998;65(4):252-8, 29-30.

13. Y M Dailey, G M Humphris, Lennon MA. A guide to care for anxious and phobic dental patients. Brit Dent J. 2001(190):450 - 3. 
14. Al-Namankany A, Ashley P, Petrie A. The development of a dental anxiety scale with a cognitive component for children and adolescents. Pediatr Dent. 2012;34(7): e219-24.

15. Buchanan H, Niven N. Validation of a Facial Image Scale to assess child dental anxiety. Int J Paediatr Dent. 2002;12(1):47-52.

16. Buchanan $\mathrm{H}$, Niven N. Further evidence for the validity of the Facial Image Scale. Int J Paediatr Dent. 2003;13(5):368-9.

17. Rãducanu AM, Feraru V, Herteliu C, Anghelescu R. Assessment of The Prevalence of Dental Fear and its Causes Among Children and Adolescents Attending a Department of Pediatric Dentistry in Bucharest. OHDMBSC. 2009; VIII (1):42-49. 18. Barros L, Buchanan $\mathrm{H}$. Correspondence between dentist and child ratings of dental anxiety in Portugal: A preliminary study. Revista Portuguesa de Estomatología, Medicina Dentária e Cirugia Maxilofacial. 2011;52(1):13-5.

19. Motta LJ, Bussadori SK, Campanelli AP, da Silva Aé L, Alfaya TA, de Godoy $\mathrm{CHL}$, et al. Pain during Removal of Carious Lesions in Children: A Randomized Controlled Clinical Trial. Int Dent J. 2013.

20. Braun V, Clarke V. Using thematic analysis in psychology. Qualitative Research in Psychology. 2006;3(2):77-101.

21. General, Dental, Council, United, Kingdom. Standards for Dental Professionals. https://wwwgdc-ukorg/Dentalprofessionals/Standards/Documents/Standards for the Dental Teampdf. 2013:10.

22. Dailey YM, Humphris GM, Lennon MA. Reducing patients' state anxiety in general dental practice: a randomized controlled trial. J Dent Res. 2002;81(5):319-22. 23. Hull, P \& Humphris, G M 2010, ' Anxiety reduction via brief intervention in dentally anxious patients: a randomized controlled trial ' Social Science and Dentistry, vol 1, no. 2, pp. 108-117.

24. Carson P, Freeman R. Assessing child dental anxiety: the validity of clinical observations. Int J Paediatr Dent. 1997 Sep 1;7(3):171-6.

25. Newton T, Asimakopoulou K, Daly B, Scambler S, Scott S. The management of dental anxiety: time for a sense of proportion? Br Dent J. 2012;213(6):271-4. 